BMJ Open Sport \& Exercise Medicine

\title{
Associations between cardiac troponin I and cardiovascular parameters after 12- week endurance training in young moderately trained amateur athletes
}

\author{
Gashaw Tesema (D) , ${ }^{1}$ Mala George ${ }^{2}$
}

\begin{abstract}
To cite: Tesema G, George M. Associations between cardiac troponin I and cardiovascular parameters after 12-week endurance training in young moderately trained amateur athletes. BMJ Open Sport \& Exercise Medicine 2021;7:e001065. doi:10.1136/ bmjsem-2021-001065
\end{abstract}

Accepted 9 March 2021

\section{Check for updates}

(C) Author(s) (or their employer(s)) 2021. Re-use permitted under CC BY-NC. No commercial re-use. See rights and permissions. Published by BMJ.

${ }^{1}$ Sport Science, Debre Tabor University, Debre Tabor, Amhara Ethiopia

${ }^{2}$ Biochemistry, Arba Minch University, Arba Minch, Southern Nations, Ethiopia

Correspondence to Dr Gashaw Tesema; gashtesema@gmail.com

\section{ABSTRACT}

Background Previous studies were conducted only on elite athletes, and they investigate acute training responses of cardiac troponin I (CTnl). However, cardiac troponin was found to be elevated in young and inexperienced athletes than adults, and immature myocardium is more susceptible to injury, which needs further consideration. Aim Therefore, we aimed to observe the association between CTnl and cardiovascular parameters in response to chronic endurance training adaptation in young athletes. Methods Fifteen participants aged $(19.5 \pm 1.3)$ years were selected and placed in endurance running at $70 \%$ 80\% HRmax intensity for 35 min per training for the first week and additional 2 min each week from the second to the last week for 12 weeks. Serum cardiac troponin and cardiovascular parameters were assessed at pre-training and after 12 weeks of training.

Result We find a significant CTnl level $(p<0.05)$ and it is positively correlated with systolic blood pressure (BP) $(r=0.425)$. Moreover, CTnl was statistically significant $(p<0.01)$ and positively associated with mean arterial pressure $(\mathrm{r}=0.516)$ with a moderate correlation. Besides, CTnl showed a significant $(p<0.001)$ and positive relationship with resting heart rate $(r=0.605)$ and a moderate correlation. We did not find a significant relationship between CTnl and diastolic BP in response to endurance training adaptation.

Conclusion In conclusion, serum CTnl was significantly and positively associated with cardiovascular parameters in young amateur athletes in response to 12-week endurance training adaptation.

\section{INTRODUCTION}

Regular endurance training decreases cardiovascular disease and related problems ${ }^{1}$ through maintaining normal blood pressure (BP) and decreasing high BP. ${ }^{2}$ Recent studies reported elevated cardiac troponin I (CTnI) levels which have been observed in marathon runners with exercise-induced high $\mathrm{BP}^{3}$ Even though cardiac troponin is found to be elevated in various clinical settings, that is, myocardial infarction, heart failure, hypertensive cases, ${ }^{4}$ recent studies reported that CTnI could be released due to myocardial

\section{Key messages}

What are the findings?

- Elevated cardiac troponin I (CTnl) levels have been observed in young athletes with exercise-induced high blood pressure (BP) after acute endurance running.

- After a 12-week chronic endurance training, CTn showed a decreased concentration and moderate positive association with systolic BP, mean arterial pressure and resting heart rate.

How might it impact clinical practice in the future?

- An elevation of serum CTnl concentration after an acute endurance training might indicate the athletes' younger age and lesser training experience. This, however, could be adaptable after chronic training.

ischaemia during exercise as a result of rapid atrial pacing in the absence of cell necrosis. ${ }^{45}$ Conversely, exercise-induced stress has shown an adverse effect on the heart by enhancing apoptosis and dysregulation of the matrix metalloproteinase system in a rat model. ${ }^{6}$ Previous studies were conducted only on elite athletes and investigated responses of CTnI with acute responses of endurance exercise with the variability of exercise-induced hypertension. However, cardiac troponin was found to be elevated in adolescents than adults ${ }^{7-9}$ after endurance training, and young myocardium may be more susceptible to injury. ${ }^{10}$ Thus, the increased interest of involvement in endurance sports by young athletes ${ }^{11}$ has driven us to observe CTnI and cardiovascular parameters.

Studies reported a transient systolic and diastolic abnormality ${ }^{12} 13$ hypertension and abnormally elevated level of cardiac troponin after endurance exercise, ${ }^{14-16}$ indicating a positive correlation between cardiac troponin elevation and the level of cardiac abnormalities. The risk of developing this abnormality 
is partly explained by the training experiences of the athletes. ${ }^{17} 18$ Moreover, systolic BP was reported to have positive associations with troponin I serum levels ${ }^{1920}$ with the acute endurance training response. However, the increase in cardiac troponin after endurance training is likely due to the cytosolic release of the biomarker, not due to the myocyte's true breakdown that does not lead to cardiovascular problems. ${ }^{21}$ Although some studies tried to observe the relationship between cardiac biomarkers and BP, they did not consider youngsters with gradual training adaptation.

Therefore, it is of interest to observe the association of cardiac troponin and cardiovascular parameters (resting heart rate (RHR), MAP, systolic and diastolic BP) in response to chronic endurance training in young athletes. Therefore, we aimed to observe the relationship between serum cardiac troponin I and cardiovascular parameters in response to long-term endurance running in young, moderately trained athletes. Accordingly, we applied continuous endurance running with $70 \%-80 \%$ HRmax intensity for $35 \mathrm{~min}$ per session for the first week and additional 2 min each week from the second to the last week for 12 weeks. Hence, we hypothesised that cardiac troponin I is significantly and positively associated with cardiovascular parameters in response to endurance training adaptation. As a result, this study will give active individuals and amateur athletes information to monitor their progress and maintain their cardiovascular health to reduce the potential of a cardiac problem and the probability of illness.

\section{METHODS}

\section{Study area}

This study was done in Bahir Dar, Ethiopia, at an altitude of $1840 \mathrm{~m}$ above the sea level with $11^{\circ} 36^{\prime} \mathrm{N} 37^{\circ} 23^{\prime} \mathrm{E}$ coordinates of latitude and longitude. It is characterised by a $25^{\circ} \mathrm{C}-32^{\circ} \mathrm{Cannual}$ average temperature and $58 \%$ annual average humidity. ${ }^{22}$

\section{Study population}

Fifteen healthy, moderately trained male athletes aged $(19.5 \pm 1.3)$ years were selected. The sample size was based on similar studies done before ${ }^{23}$ using the following equations, which consider $80 \%$ power and $\mathrm{p} \leq 0.05$ :

$n=\frac{(z \alpha+z \beta / 2)^{2}(\sigma 2+\sigma 2) R * T *}{(\delta-/ C V T-C V R /)^{2}}, \quad n=\frac{(1.64+0.84)^{2}\left(0.30^{2}+0.35^{2}\right)}{(0.10+0.70-0.50)^{2}}=14.5$

where $n=$ sample size needed per group, $\sigma^{* 2} \mathrm{~T}$ is the variance of the treatment, $\sigma^{* 2} \mathrm{R}$ is the variance of the reference, $\delta$ is the maximum percent of no clinical importance, CVT is the coefficient of variations of the treatment and CVR is the coefficient of variations of the reference.

In addition, a validated physical activity readiness questionnaire $^{24}$ was given to evaluate conditions that may exclude participants from participating in endurance running. Therefore, participants that reported health problems (ie, heart dysfunction and chest pain during exercise), physical conditions (ie, loss of balance, consciousness, and bone or joint problem) and currently taking antihypertensive drugs were excluded from the study. In addition, participants who were smokers and alcoholics were also excluded from participation in the study due to effects on both physical performance and metabolic biomarkers. ${ }^{25} 26$

\section{Study design}

A single group pre-post study design was used in this study. Fifteen subjects were selected and placed in highintensity endurance training. Serum cardiac troponin and cardiovascular parameters were assessed at the beginning of the training and after 12 weeks of endurance training. High-intensity endurance training was done at $70 \%-80 \%$ HRmax 35 min per session for the first week and 2-minute increments each week from the second to the last week for 12 weeks.

Written consent was delivered to the participants, and they were informed about the objectives of the study. Participation of subjects in this study was purely voluntary, and their right not to participate was respected. Issues of confidentiality and anonymity were maintained, too.

\section{Training protocol}

This field-based study was conducted based on the protocol specified here. There were 5 min of warming up and stretching exercises at the beginning of the training and 5 min of cooling down exercise at the end of each training session. Then continuous endurance running was done at $70 \%-80 \%$ HRmax at a constant intensity of 35 min per session for the first week and 2-minute increments each week from the second to the last week for 12 weeks. Participants were provided with heart rate (HR) monitors, and the training intensity was monitored with a Polar HR monitor (Polar Electro Oy, Kempele, Finland) throughout the entire session. Intensities were individualised by adjusting training paces based on the percent maximal HR (\% HRmax). ${ }^{27}$ They were instructed not to participate in other exercise training and continue with their nutrition habits throughout the experiment.

\section{Measurements of cardiovascular parameters}

Systolic and diastolic BP were measured with an electronic BP monitor (Spengler ES-60, France). The tourniquet of the sphygmomanometer was tied on the wrist of the lower arm and inflated with air while the subject was seated. The result displayed from the indicator of the sphygmomanometer was recorded in millimetre mercury. Mean arterial pressure (MAP) was computed using the following formula: $\mathrm{MAP}=\mathrm{DBP}+1 / 3(\mathrm{SBP}-\mathrm{DBP}){ }^{28}$

Measurement of RHR was done in the morning immediately after waking up using a HR monitor. The HR monitor sensor was tied on the hand's radial artery, and the subject laid down relaxed in a supine position. 
After about $1 \mathrm{~min}$, the HR monitor was started. The subject breathed calmly for $3-5$ min without looking at the HR monitor. Then after 3-5 min, the HR monitor was stopped. Finally, the result of HR was checked and recorded.

\section{Assay of serum CTnl}

About $5 \mathrm{~mL}$ of blood samples was collected just before training (pretest) and 4 hours after the 12-week posttraining sessions from each participant. The peak of cardiometabolic markers has been observed 3-4hours after the training period. $^{729}$ The blood was withdrawn from an antecubital vein with vacutainer serum separator tube containing blood clotting accelerant gel. The serum was isolated by centrifugation of the blood sample at $4000 \mathrm{rpm}$ (revolution per minute) for $3 \mathrm{~min}$ and stored at $-20^{\circ} \mathrm{C}$ until analysis. The amount of cardiac troponin I band (cTnI) was measured by chemiluminescence immunoassay using Maglumi 800 fully automated chemiluminescence immunoassay analyser in $\mathrm{ng} / \mathrm{mL}$ via Shenzhen New Industries Biomedical Engineering (Snibe).

\section{Data analysis}

To analyse the data found from the study, inferential statistics were used. The Kolmogorov-Smirnov and Shapiro-Wilk ${ }^{30} 31$ normality tests have been applied to check whether the data are normally distributed. To identify the presence of any significant correlations between variables, Pearson's correlation analysis was applied. IBM-SPSS V.20 packages were used to analyse the data. Variables were specified as Pearson's correlation coefficient (r) and probability value (p). In all cases we took, $\mathrm{p} \leq 0.05$ was reported as being a significant result.

\section{Patient and public involvement}

Patients and/or the public were not involved in the design, or conduct, or reporting or dissemination plans of this research.

\section{RESULTS}

Based on Kolmogorov-Smirnov and Shapiro-Wilk normality tests, ${ }^{3031}$ we find $\mathrm{p}>0.05$. Also, visual inspection of histograms, normal Q-Q plots and box plots displayed that the data were approximately normally distributed. The general characteristics of participants in mean $\pm \mathrm{SD}$ were age $(19.5 \pm 1.3)$ years, height $(1.67 \pm 0.07) \mathrm{m}$, body mass $(53.96 \pm 5.67) \mathrm{kg}$ and body mass index $(19.31 \pm 1.41)$ $\mathrm{kg} / \mathrm{m}^{2}$.

After the 12-week endurance training, serum cardiac troponin was found to be correlated with cardiovascular parameters (systolic BP, MAP and RHR) (table 1). The level of CTnI after the 12-week endurance training was significantly $(\mathrm{p}<0.05)$ and positively associated with systolic BP $(\mathrm{r}=0.425)$, indicating a weak relationship. Moreover, CTnI was statistically significant $(\mathrm{p}<0.01)$ and positively associated with MAP $(\mathrm{r}=0.506)$ with a moderate relationship. Furthermore, CTnI showed a significant $(\mathrm{p}<0.001)$ and positive association with RHR $(\mathrm{r}=0.605)$ with a moderate relationship. In both cases, CTnI and cardiac parameters were found to be decreased at the end of the 12-week endurance training. However, we did not find a significant association between CTnI and diastolic BP after the 12-week endurance training adaptation in young, moderately trained athletes.

\section{DISCUSSION}

This study aimed to look for the relationship between serum cardiac troponin I and cardiovascular parameters in response to the 12-week endurance training in young athletes. The study's main findings support our hypothesis that serum cardiac troponin I is positively associated with cardiovascular parameters after endurance training adaptation. Consequently, we find a reduced serum cardiac troponin $(\mathrm{CTnI})$ in response to the 12-week endurance training, indicating a significant and positive correlation with RHR $(\mathrm{p}<0.001)$, MAP $(\mathrm{p}<0.01)$ and systolic BP $(p<0.05)$. However, we did not find a

\begin{tabular}{|c|c|c|c|c|c|c|}
\hline & & CTnl & SYSTBP & MAP & RHR & DIASTBP \\
\hline \multirow[t]{2}{*}{ CTnl } & Pearson's $r$ & _- & & & & \\
\hline & $P$ value & _- & & & & \\
\hline \multirow[t]{2}{*}{ SYSTBP } & Pearson's $r$ & $0.425^{\star}$ & - & & & \\
\hline & $P$ value & 0.019 & _- & & & \\
\hline \multirow[t]{2}{*}{ MAP } & Pearson's r & $0.516^{*}$ & $0.586^{* * *}$ & - & & \\
\hline & $P$ value & 0.004 & $<0.001$ & _- & & \\
\hline \multirow[t]{2}{*}{ RHR } & Pearson's r & $0.605^{\text {*k* }}$ & 0.285 & $0.540^{* *}$ & - & \\
\hline & $P$ value & $<0.001$ & 0.127 & 0.002 & _ & \\
\hline \multirow[t]{2}{*}{ DIASTBP } & Pearson's r & 0.198 & 0.029 & $0.432^{*}$ & 0.178 & - \\
\hline & $P$ value & 0.294 & 0.878 & 0.017 & 0.347 & _ \\
\hline
\end{tabular}

${ }^{*} p<0.05 ;{ }^{* *} p<0.01 ;{ }^{* * *} p<0.001$.

CTnl, cardiac troponin I band; DIASTBP, diastolic blood pressure; MAP, mean arterial pressure; RHR, resting heart rate; SYSTBP, systolic blood pressure. 
significant association between CTnI and diastolic BP $(p>0.05)$ in response to the 12-week endurance training adaptation.

This study confirmed a reduced and significant positive association between CTnI and systolic BP in response to the 12-week endurance training. In line with our study, systolic BP was reported to have positive associations with troponin I serum levels. ${ }^{19}{ }^{20}$ Conversely, an abnormally elevated cardiac troponin level related to high BP with positive association following acute endurance exercise has been reported. ${ }^{14-16}$ This abnormality could be explained by the training experiences of the athletes. ${ }^{1718}$

Different studies reported a significant reduction of $\mathrm{MAP}^{32}$ and systolic BP in response to chronic endurance training, indicating reduced $\mathrm{BP}^{33}{ }^{34}$ These reports support our finding that MAP was significantly reduced and was positively associated with cardiac troponin. This could be attributed to the decreased concentration of plasma endothelin-1 (ET-1), a potent vasoconstrictor peptide produced by vascular endothelial cells, ${ }^{35}$ resulting in decreased $\mathrm{BP}$ and associated cardiac troponin in response to endurance training adaptation. Accordingly, the reduction in the cardiac troponin release in experienced endurance athletes might be explained by myocardial scar replacing with new and normal cardiac tissue.

In this study, we confirmed a significantly reduced and positively correlated resting heart rate with cardiac troponin. Even though we did not find similar studies observing RHR association with cardiac troponin, different studies reported that RHR was reduced after 8-12 weeks of high-intensity endurance training. ${ }^{32} 36$ The reduction of these cardiac parameters could be attributed to altered autonomic regulation of HR towards vagal dominance $^{36}$ and lower sympathetic and increased parasympathetic outflow, increased muscular capillarisation, vascular remodelling with a resulting reduction in systemic vascular resistance. ${ }^{37}$ Changes in serum cardiac troponin can be influenced by training experience, intensity differences and training method differences that we could not address in our study design. Nevertheless, we can see a chronic endurance training effect with a gradual increment in the training duration for 12 weeks. Generally, our findings improve the understanding of physiological and pathological foundations of serum CTnI association with cardiovascular parameters after long-term endurance training in young athletes. In addition, these findings add to the growing body of evidence linking cardiac troponin to cardiovascular fitness. This indicates that gradual training adaptation reduces serum cardiac troponin concentration and associated cardiovascular parameters after the 12-week endurance training in young, moderately trained amateur athletes. However, further investigation is needed across different intensity and different methods of training.

\section{CONCLUSION}

In this study, we concluded that serum CTnI is significantly and positively associated with cardiovascular parameters in amateur athletes in response to the 12-week endurance training.

Contributors GT contributed to the conception and design of the study, analysis, or interpretation of data for the work; MG drafted the study and revised it critically for important intellectual content and final approval of the manuscript to be published.

Funding The authors have not declared a specific grant for this research from any funding agency in the public, commercial or not-for-profit sectors.

Competing interests None declared.

Patient consent for publication Not required.

Ethics approval Ethical approval was obtained from the Institutional Research Ethics Review Committee of Mekelle University conformed to the 1975 Declaration of Helsinki.

Provenance and peer review Not commissioned; externally peer reviewed.

Data availability statement All the data relevant to this study is incorporated to this manuscript.

Open access This is an open access article distributed in accordance with the Creative Commons Attribution Non Commercial (CC BY-NC 4.0) license, which permits others to distribute, remix, adapt, build upon this work non-commercially, and license their derivative works on different terms, provided the original work is properly cited, appropriate credit is given, any changes made indicated, and the use is non-commercial. See: http://creativecommons.org/licenses/by-nc/4.0/.

\section{ORCID iD}

Gashaw Tesema http://orcid.org/0000-0001-8962-2313

\section{REFERENCES}

1 Lee HY, Kim JH, Kim BO, et al. Regular exercise training reduces coronary restenosis after percutaneous coronary intervention in patients with acute myocardial infarction. Int $J$ Cardiol 2013;167:2617-22.

2 Ruivo JA, Alcântara P. [Hypertension and exercise]. Rev Port Cardiol 2012;31:151-8.

$3 \mathrm{Kim}$ YJ, Shin YO, Lee YH, et al. Effects of marathon running on cardiac markers and endothelin-1 in EIH athletes. Int J Sports Med 2013;34:777-82.

4 White HD. Pathobiology of troponin elevations: do elevations occur with myocardial ischemia as well as necrosis? J Am Coll Cardiol 2011:57:2406-8.

5 Turer AT, Addo TA, Martin JL, et al. Myocardial ischemia induced by rapid atrial pacing causes troponin $T$ release detectable by a highly sensitive assay: insights from a coronary sinus sampling study. J Am Coll Cardiol 2011;57:2398-405.

6 Oláh A, Németh BT, Mátyás C, et al. Cardiac effects of acute exhaustive exercise in a rat model. Int J Cardiol 2015;182:258-66.

7 Tian Y, Nie J, Huang C, et al. The kinetics of highly sensitive cardiac troponin $\mathrm{T}$ release after prolonged treadmill exercise in adolescent and adult athletes. J Appl Physiol 2012;113:418-25.

8 Shave R, Baggish A, George K, et al. Exercise-Induced cardiac troponin elevation: evidence, mechanisms, and implications. J Am Coll Cardiol 2010;56:169-76.

9 Niemelä M, Kangastupa P, Niemelä O, et al. Individual responses in biomarkers of health after marathon and half-marathon running: is age a factor in troponin changes? Scand J Clin Lab Invest 2016;76:575-80.

10 Kannankeril PJ, Pahl E, Wax DF, Wd PE. Usefulness of troponin I as a marker of myocardial injury after pediatric cardiac catheterization. Am J Cardiol 2002;90:1128-32.

11 Roberts WO. Can children and adolescents run Marathons? Sports Med 2007;37:299-301.

$12 \operatorname{Re} \mathrm{S}$, Dawson E, Whyte G, et al. Evidence of exercise-induced cardiac dysfunction and elevated CTnT in separate cohorts competing in an ultra-endurance mountain marathon race. Int $J$ Sport Med 2002;23:489-94.

13 Dávila-Román VG, Guest TM, Tuteur PG, et al. Transient right but not left ventricular dysfunction after strenuous exercise at high altitude. $J$ Am Coll Cardiol 1997;30:468-73. 
14 La Gerche A, Connelly KA, Mooney DJ, et al. Biochemical and functional abnormalities of left and right ventricular function after ultra-endurance exercise. Heart 2008;94:860-6.

15 Neilan TG, Januzzi JL, Lee-Lewandrowski E, et al. Myocardial injury and ventricular dysfunction related to training levels among nonelite participants in the Boston marathon. Circulation 2006;114:2325-33.

16 Vidotto C, Tschan $\mathrm{H}$, Atamaniuk J, et al. Responses of $\mathrm{N}$-terminal pro-brain natriuretic peptide (NT-proBNP) and cardiac troponin I (cTnl) to competitive endurance exercise in recreational athletes. Int J Sports Med 2005;26:645-50.

17 Scharhag J, George K, Shave R, et al. Exercise-associated increases in cardiac biomarkers. Med Sci Sports Exerc 2008;40:1408-15.

18 López-Laval I, Legaz-Arrese A, George K, et al. Cardiac troponin I release after a basketball match in elite, amateur and junior players. Clin Chem Lab Med 2016;54:333-8.

19 Nunes JPL, Mota Garcia JM, Farinha RMB, et al. Cardiac troponin I in aortic valve disease. Int $J$ Cardiol 2003;89:281-5.

20 Kim Y-J, Ahn JK, Shin K-A, et al. Correlation of cardiac markers and biomarkers with blood pressure of middle-aged marathon runners. $J$ Clin Hypertens 2015;17:868-73.

21 Mousavi N, Czarnecki A, Kumar K, et al. Relation of biomarkers and cardiac magnetic resonance imaging after marathon running. $A m \mathrm{~J}$ Cardiol 2009;103:1467-72.

22 Haile Z. Assessment of climate change impact on the net basin supply of lake tana water balance. The Netherlands International institute for geo- information science and earth observation ENSCHEDE; 2009.

23 Sivaraman P. Effect of eight weeks aerobic exercises on physical and physiological variables among College men. Int J Phys Educ Fit Sport 2014;3:113-7.

24 Der W, Vk J. The physical activity readiness questionnaire for everyone (PAR-Q+) and electronic physical activity readiness medical examination (ePARmed-X+). Heal Fit J Canada 2011;4:3-23.

25 Barnes MJ. Alcohol: impact on sports performance and recovery in male athletes. Sports Med 2014;44:909-19.
26 Lee C-L, Chang W-D. The effects of cigarette smoking on aerobic and anaerobic capacity and heart rate variability among female university students. Int J Womens Health 2013;5:667-79.

27 Seiler S, Stephen S. What is best practice for training intensity and duration distribution in endurance athletes? Int J Sports Physiol Perform 2010;5:276-91.

28 McArdle WD, Katch FI, Katch VL. Exercise physiology: energy, nutrition and human performance. 7th edition. Baltimore: Williams \& Williams, 2010.

29 Legaz-Arrese A, López-Laval I, George K, et al. Impact of an endurance training program on exercise-induced cardiac biomarker release. Am J Physiol Heart Circ Physiol 2015;308:H913-20.

30 Razli M, Yap W. Power comparisons of some selected normality tests. Proceedings of the Regional Conference on Statistical Sciences, 2010:126-38.

31 Shapiro SS, WILK MB. An analysis of variance test for normality (complete samples). Biometrika 1965;52:591-611.

32 Connolly LJ, Bailey SJ, Krustrup P, et al. Effects of self-paced interval and continuous training on health markers in women. Eur $J$ Appl Physiol 2017; 117:2281-93.

33 Kenney MJ, Seals DR. Postexercise hypotension. key features, mechanisms, and clinical significance. Hypertension 1993:22:653-64.

34 Raine NM, Cable NT, George KP, et al. The influence of recovery posture on post-exercise hypotension in normotensive men. Med Sci Sports Exerc 2001;33:404-12.

35 Maeda S, Tanabe T, Miyauchi T, et al. Aerobic exercise training reduces plasma endothelin-1 concentration in older women. $J$ Appl Physiol 2003;95:336-41.

36 Tulppo MP, Hautala AJ, Mäkikallio TH, et al. Effects of aerobic training on heart rate dynamics in sedentary subjects. J Appl Physiol 2003;95:364-72.

37 Andersen LJ, Randers MB, Westh K, et al. Football as a treatment for hypertension in untrained 30-55-year-old men: a prospective randomized study. Scand J Med Sci Sports 2010;20 Suppl $1: 98-102$ 